\title{
DISPLACEMENTS OF TROCHANTERIC FRACTURES AND THEIR INFLUENCE ON REDUCTION
}

\author{
J. M. B. May and P. B. Chacha, Glasgow, Scotland \\ From the Western Infirmary, Glasgow
}

The management of trochanteric fractures of the femur presents fewer problems than that of transcervical fractures. Union is often well advanced at twelve to fourteen weeks, by which time most patients are permitted to bear weight through the injured hip; the functional end-result is generally good, providing there is reasonable anatomical alignment of the fragments.

Most surgeons use internal fixation in the management of this injury, and the greater part of the literature is concerned with the merits of various fixation devices and the complications resulting from their use. A clear description of the dynamics of the displacement of the fragments was given by Horn and Wang (1964), based on the level of the fracture and the relevant muscle anatomy. This information helps to indicate the methods of reduction that should be employed.

Although an imperfect reduction does not materially alter the eventual function of the hip it does increase the difficulties of fixation. In such circumstances fixation of the fracture is not sound and the risk of complications such as bending and breaking of the nail-plate and extrusion of the nail is considerably increased.

This paper describes the detailed radiographic appearances of the displaced fragments and their relevance in achieving more precise reduction. Crack fractures and those with only slight displacement present no problems and are not considered in this discussion.

\section{MATERIALS AND METHODS}

The radiographs of 100 patients treated between 1962 and 1963 were selected at random. All the patients had been operated on and the fracture fixed by a fixed-angle nail-plate. Radiographs of the fracture taken before operation were studied and an attempt was made to determine the relative positions of the proximal and distal fragments. Post-reduction radiographs and those after operation and at follow-up were scrutinised in the same manner and note was taken of any difficulty experienced in reducing the fracture or inserting the nail-plate.

\section{DISPLACEMENT OF FRAGMENTS}

This study has revealed two types of trochanteric fracture which are of practical significance. In Type I fractures the proximal fragment of the femur consists of the head and neck alone; in Type II fractures the head, neck and a major part of the greater trochanter constitute the proximal fragment.

In the first type the proximal fragment, having no muscle attachment, remains in a neutral position, except in the slightly displaced fracture when it rotates laterally in continuity with the distal fragment under the influence of gravity.

In the second type the lateral rotator muscles of the hip that are inserted into the greater trochanter tend to rotate the proximal fragment laterally (Horn and Wang 1964). This will occur, however, only if the two main fragments are not impacted and if the soft tissues crossing the main fracture site are disrupted. Even if the greater trochanter is fractured it may remain a functional part of the proximal fragment by the preservation of its soft-tissue attachments -in particular the retinaculum of Weitbrecht, consisting of fibres of the posterior capsule reflected proximally on to the femoral neck. Discussing the role of this retinaculum 
in transcervical fractures of the femur, Smith (1953) likened it to the binding of a book; and in a similar context Garden (1961) stressed the importance of its presence in the reduction

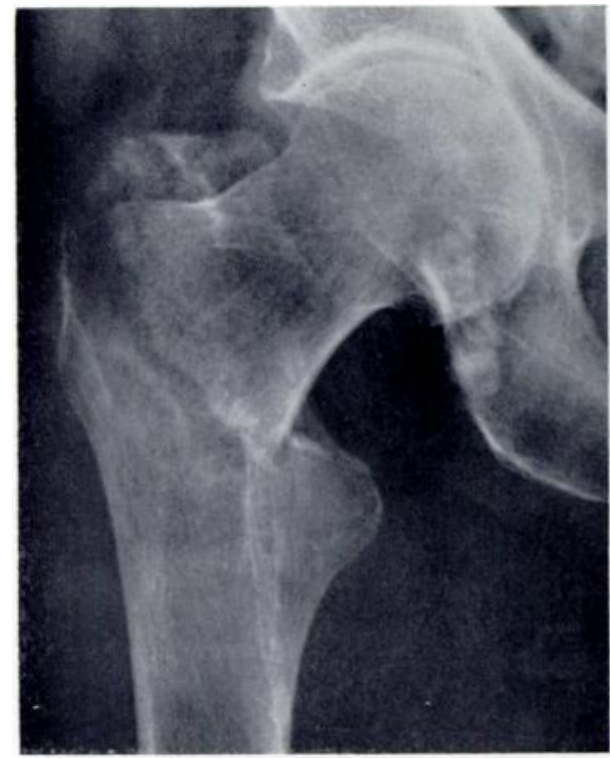

FIG. 1

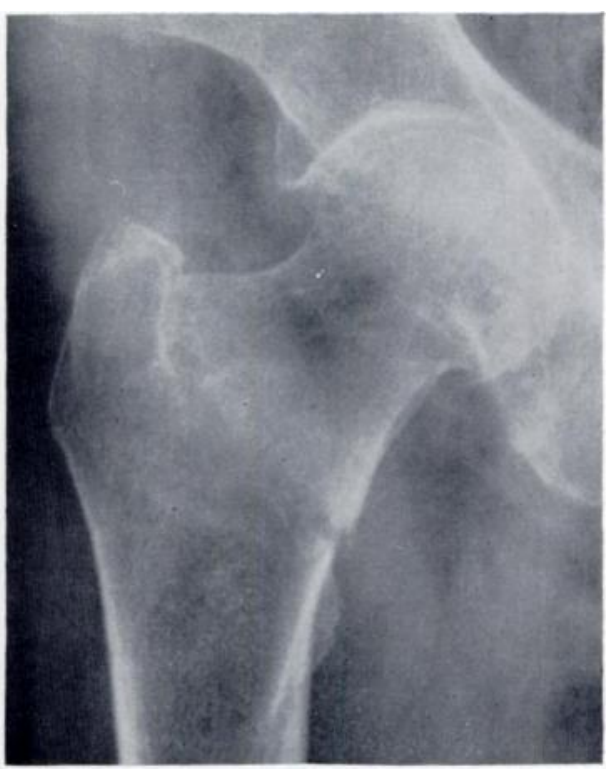

FiG. 2

Figure 1-The antero-posterior radiographs of a Type I fracture. Figure 2-After manipulation of the lower limb into medial rotation: in this patient the excessive medial rotation was not detrimental as there must have been a soft-tissue hinge posteriorly, which allowed the fracture to remain closed.

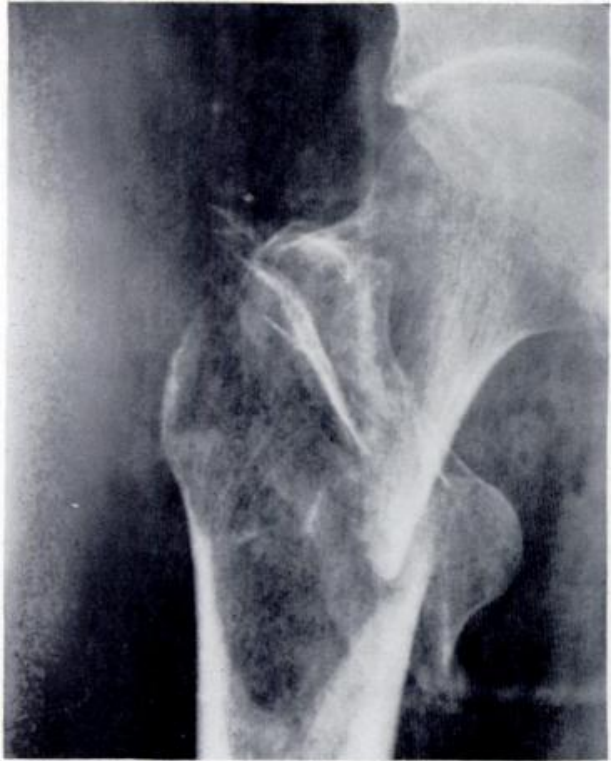

FiG. 3

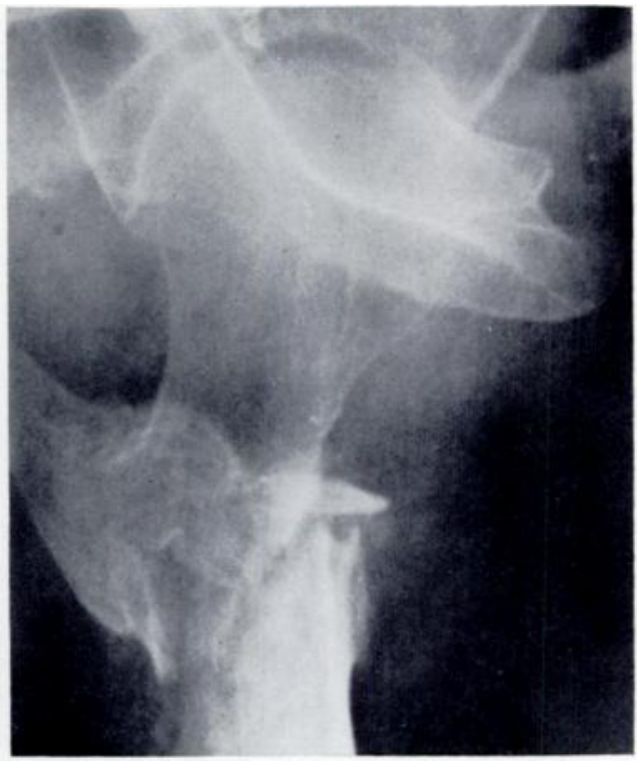

Fig. 4

Figure 3-Antero-posterior radiograph of a Type II fracture taken with the lower limb in medial rotation, showing foreshortening of the femoral neck. Figure 4 Lateral radiograph showing a winged appearance of the greater trochanter with posterior inclination of the axis of the femoral neck.

of fractures of the femoral neck. Because of the natural tendency for the lower limb to roll outwards in fractures around the hip, independent lateral rotation of the proximal fragment only becomes obvious when the distal fragment is rotated medially.

VOL. $50 \mathrm{~B}$, NO. 2, MAY 1968 
The distal fragment tends to rotate laterally when a fracture occurs; in addition, posterior displacement will occur from the effect of gravity if the soft tissues are sufficiently disrupted. This may happen in either of the types of fracture described.

Both types of fracture may be associated with varus at the fracture site. Associated fractures of the lesser trochanter and calcar femorale do not actively influence the displacement of the main fragments.

\section{RADIOLOGICAL APPEARANCES}

Recognition of the position of the proximal fragment is essential to ensure accurate reduction. Initial radiographs are always taken when the limb is rolled outwards by gravity. The position of the proximal fragment, independent of the distal fragment, can only be seen when radiographs are taken with the limb in the neutral position.

Type I fracture-The initial antero-posterior radiograph in this fracture shows that the neck of the femur is not foreshortened. The greater trochanter is either a part of the distal fragment or it is widely separated from both main fragments; its outline may be seen superimposed on the shadow of the femoral neck (Fig. 1). When the distal fragment is rotated into the neutral position the femoral neck remains unchanged; the greater trochanter moves with the distal fragment or remains behind, depending on its relationship to it (Fig. 2).

In the initial lateral radiographs the axis of the femoral neck is in a neutral position, and the greater trochanter presents a "winged" appearance, either because it is a part of a laterally rotated distal fragment or because, when fractured, it is pulled there by the lateral rotator muscles which are attached to it. Lateral rotation of the distal fragment is also responsible for the anterior angulation at the fracture site. Bringing the injured limb into the neutral position corrects the angulation, and the winged appearance of the greater trochanter may or may not disappear, depending on its relation to the distal fragment.

Type II fracture-Lateral rotation of the proximal fragment is recognised by the appearance of the femoral neck and the greater trochanter on antero-posterior and lateral radiographs, when the limb is in the neutral position.

In the antero-posterior view there is foreshortening of the femoral neck, on the outline of which is superimposed that of the greater trochanter behind it (Fig. 3). The lateral radiograph reveals a "winged" appearance of the greater trochanter with posterior inclination of the axis of the femoral neck (Fig. 4).

In this analysis Type I fractures accounted for 80 per cent and Type II fractures for 20 per cent of the total number studied.

\section{ROLE OF THE GREATER TROCHANTER}

When the greater trochanter is not fractured it is easy to recognise whether it belongs to the proximal or to the distal fragment. However, fractures of the greater trochanter are often present and it is important to note the features that will determine its relationship to the main fragments.

In some Type II fractures the greater trochanter is avulsed and rotated backwards on a posterior soft-tissue hinge of joint capsule (Weitbrecht's retinaculum) (Figs. 5 and 6). In these cases the pull of the lateral rotator muscles is not as effective as it would be with an intact greater trochanter, but it may be sufficient to produce some lateral rotation deformity of the proximal fragment. The antero-posterior radiograph will show a slightly foreshortened femoral neck with marked superimposition of the outline of the greater trochanter. The lateral view will show some posterior inclination of the neck of the femur and an obvious " winged" appearance of the greater trochanter.

It is not unusual for the greater trochanter to be avulsed completely from both main fragments and pulled backwards by the lateral rotator muscles. In such cases no effective soft-tissue continuity is retained with the proximal fragment, which remains in the neutral position. The radiological appearances of this Type I fracture will be recognised with certainty 
only when the distal fragment is disengaged and placed in the neutral position. The anteroposterior radiograph shows a femoral neck in the neutral position with a superimposed greater trochanter. On the lateral view the axis of the femoral neck is neutral but the greater trochanter is seen as a separate fragment producing a gross "winged" appearance.

From this description one can see that the greater trochanter and its fractures play a key role in influencing the displacement of the main fragments.

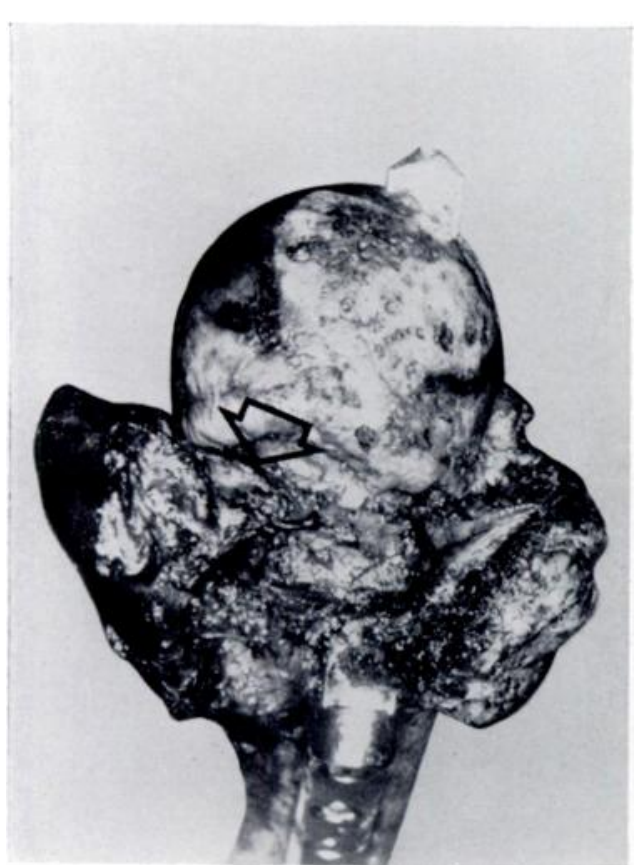

Fig. 5

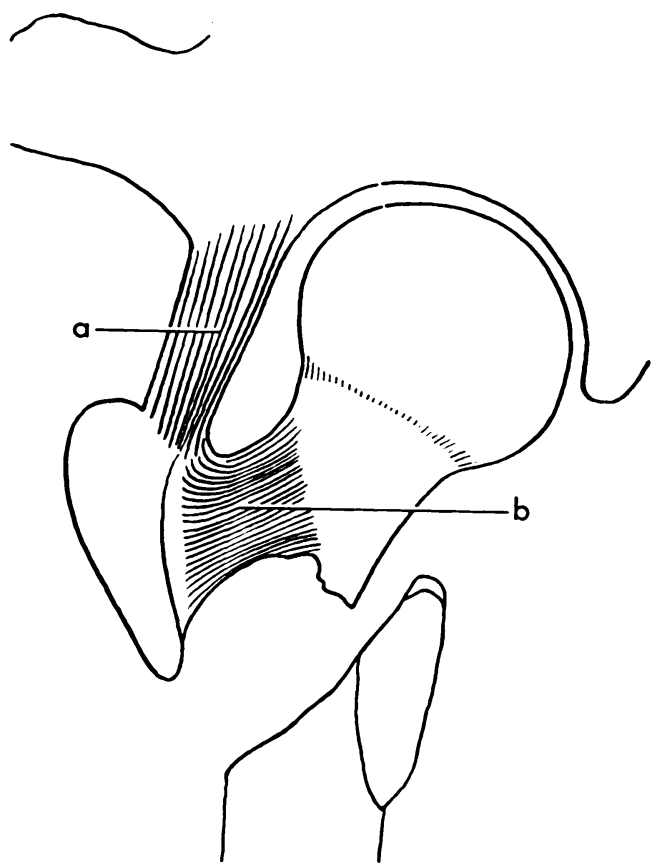

Fig. 6

Figure 5-The specimen of a trochanteric fracture, orientated as in a lateral radiograph, to show the posterior soft-tissue hinge (arrowed) between the greater trochanter and the proximal fragment. Figure 6-A drawing of Figure 5 showing the lateral rotators of the hip $(a)$; and the posterior soft-tissue hinge $(b)$.

\section{REDUCTION OF TROCHANTERIC FRACTURES}

The reduction of trochanteric fractures is not clearly described in the literature. Morris (1941), Evans (1949, 1951) and Massie (1964) pointed out that some fractures need neutral rotation and others lateral rotation of the distal fragment in order to achieve a reasonable reduction. Morris and Evans did not specify which of these manoeuvres is necessary for a particular fracture. Massie advocated palpation of the exposed fracture site and study of the radiographs during manipulation; but he did not describe any radiological features which enable a correct method of reduction to be chosen.

Whereas Type I fractures require rotation of the distal fragment to a neutral position, Type II fractures will reduce with the distal fragment placed in some degree of lateral rotation. This study confirms the statements by Evans and Massie that medial rotation for the reduction of trochanteric fractures is not necessary. As Evans indicated, this manoeuvre is detrimental; it produces a posterior gap and backward angulation at the fracture site. This defect may sometimes be seen in antero-posterior and lateral radiographs, and can be misinterpreted as a destructive lesion in bone (Figs. 3 and 4).

In Type II fractures difficulties may occur with the insertion of a fixation device when reduction has been attempted by medial rotation of the distal fragment. Because of the posterior angulation at the fracture, a guide wire passed from the subtrochanteric region will tend to pass through the anterior part of the femoral neck into the posterior segment of the head, and a nail-plate placed in this position will not provide adequate fixation of the fracture. 
When a Type II fracture is incorrectly manipulated by medial rotation of the limb the antero-posterior radiograph may present a false appearance of reduction. Although the fragments appear well opposed they are only in contact along their anterior edges; the lateral

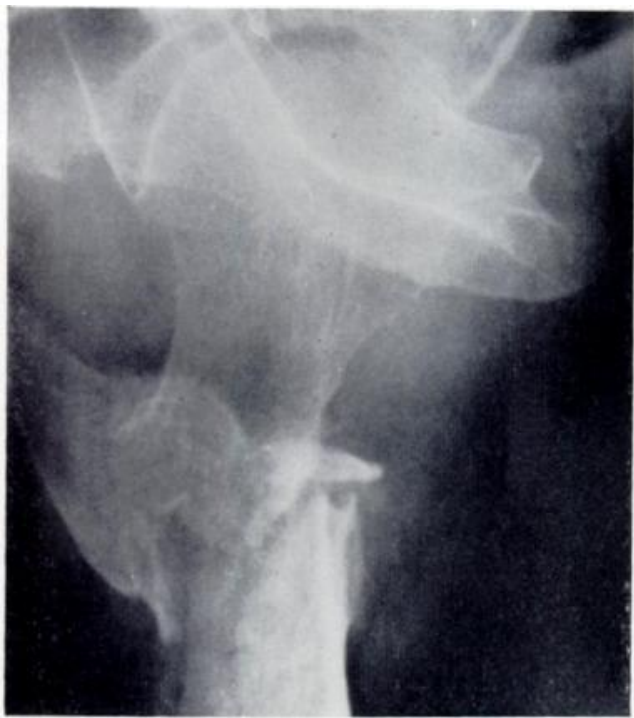

FIG. 7

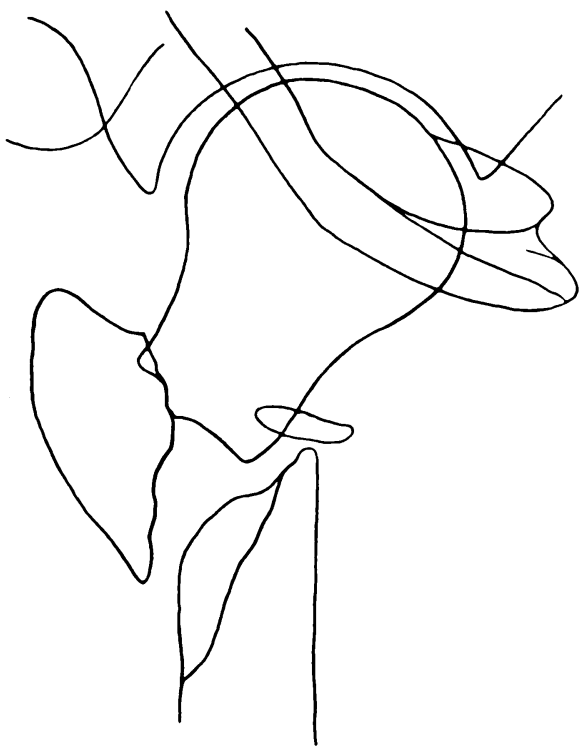

FIG. 8

Figure 7-The lateral radiograph. Figure 8-A drawing of a Type II fracture showing the posterior defect after medial rotation of the distal fragment.

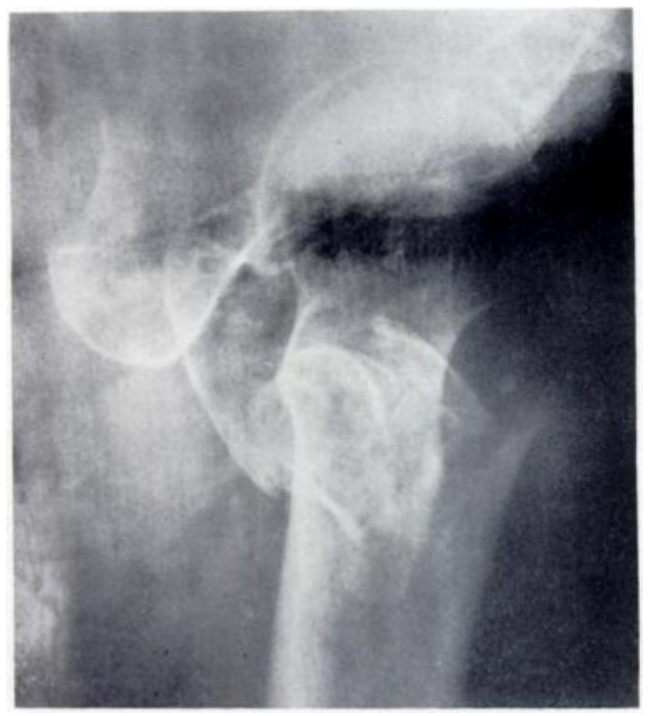

Fig. 9

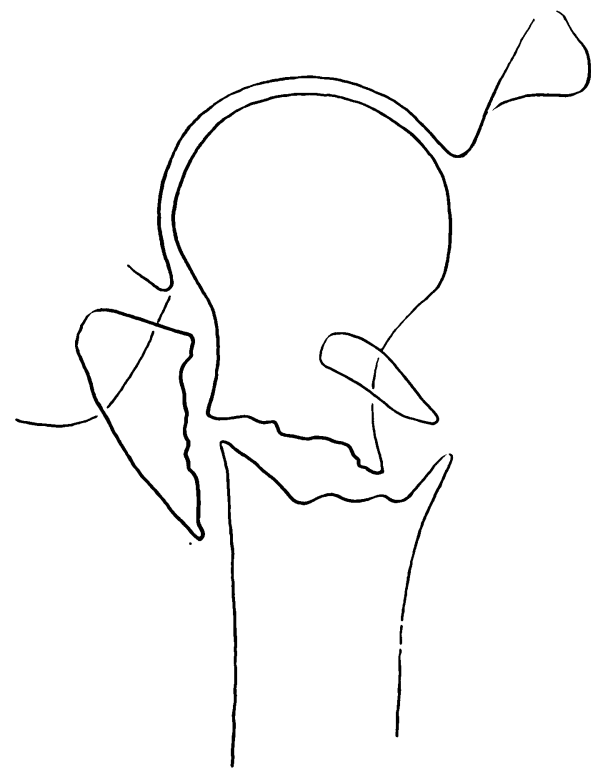

Fig. 10

Figure 9-The original lateral radiograph of the fracture in Figures 7 and 8. Figure 10-A drawing showing the absence of a defect when both fragments are lying in lateral rotation.

radiograph will reveal what appears to be a posterior defect which is due to the rotation at the fracture site producing angulation that opens backwards (Figs. 7 and 8). In the original lateral radiographs it is likely that no posterior defect is seen because the lower fragment also lies in lateral rotation (Figs. 9 and 10). 
A further difficulty may arise with both types of fracture when the distal fragment is displaced posteriorly. This, however, should be seen on the lateral radiograph and steps taken, during the insertion of the guide wires, to lift the femoral shaft anteriorly.

\section{CONCLUSION}

An appreciation of the relative position of the main fragments in these fractures is necessary to obtain better reduction and fixation than is often achieved.

The radiological appearances of the proximal fragment enable its independent rotation to be defined and this, in turn, permits a simple classification of all these fractures into two types, depending on the behaviour of the proximal fragment. The cause for a particular displacement is found by studying the radiographic appearance of the greater trochanter and appreciating its relationship to the proximal fragment; when it is independent of the proximal fragment, either because it is a part of the distal fragment or because of complete bone and soft-tissue separation from both the main fragments, a Type I fracture occurs. If it is a part of the proximal fragment a Type II fracture results. If the greater trochanter is fractured but retains sufficient soft-tissue connection with the head and neck of the femur, a Type II fracture also occurs, but with less marked lateral rotation of the proximal fragment.

Normally the fracture is classified on the appearance of the antero-posterior and lateral radiographs taken after the patient is anaesthetised and traction applied to the limb in the neutral position. If it is a Type I fracture, reduction should have been achieved. If it is a Type II fracture the leg is rolled into lateral rotation, which must be judged empirically from the " neutral" radiographs. After further radiographs to confirm reduction, operation can then be begun and, if necessary, precise reduction can be completed with a finger palpating the fracture site posteriorly.

\section{SUMMARY}

1. The radiological appearances of the fragments in 100 trochanteric fractures have been analysed.

2. A simple practical classification enables the correct method of reduction to be chosen.

3. In Type I fractures the proximal fragment consists of the head and neck alone. In Type II fractures the head, neck and a major part of the great trochanter constitute the proximal fragment.

4. The key role of the greater trochanter in influencing the displacement of the fragments is discussed.

5. For reduction, Type I fractures require rotation of the distal fragment to a neutral position. Type II fractures reduce in some degree of lateral rotation.

Our appreciation is due to Professor Roland Barnes for suggesting this study and for his helpful criticism in the preparation of this paper; to the surgeons of the orthopaedic department for allowing us to review radiographs of their patients; and to Mr R. Loudon Brown, Senior Medical Photographer, Glasgow South-Western Hospitals, for his valuable assistance with the illustrations.

\section{REFERENCES}

Evans, E. M. (1949): The Treatment of Trochanteric Fractures of the Femur. Journal of Bone and Joint Surgery, 31-B, 190.

Evans, E. M. (1951): Trochanteric Fractures. Journal of Bone and Joint Surgery, 33-B, 192.

GARDEN, R. S. (1961): Low-Angle Fixation in Fractures of the Femoral Neck. Journal of Bone and Joint Surgery, 43-B, 647.

HoRn, J. S., and WANG, Y. C. (1964): The Mechanism, Traumatic Anatomy and Non-Operative Treatment of Intertrochanteric Fracture of the Femur. British Journal of Surgery, 51, 574.

MASSIE, W. K. (1964): Fractures of the Hip. Journal of Bone and Joint Surgery, 46-A, 658.

MorRIS, H. D. (1941): Trochanteric Fractures. Southern Medical Journal, 34, 571.

SмIтH, L. D. (1953): Hip Fractures. Journal of Bone and Joint Surgery, 35-A, 367.

VOL. 50 B, NO. 2, MAY 1968 